\title{
Competencias didácticas en cursos online de posgrado universitario
}

\author{
María Julia GonZÁLEz CondE \\ mariajul@ccinf.ucm.es \\ Universidad Complutense de Madrid \\ Carmen SAlgado SAnTAmaría \\ carmen.salgado@ccinf.ucm.es \\ Universidad Complutense de Madrid \\ Javier FoMBONA CADAVIECO \\ fombona@uniovi.es \\ Universidad de Oviedo
}

Recibido: 13/07/2012

Aceptado: 22/10/2012

\section{Resumen}

El objetivo de este artículo es analizar las técnicas y competencias principales que precisan los docentes en los cursos no presenciales de Posgrado. Una oferta educativa que empieza a incrementarse con gran fuerza en la mayoría de las instituciones universitarias más prestigiosas y cuya base experimental se halla en una fase de inicio y acertada consolidación. Hemos examinado los nuevos métodos en cuanto a su forma y contenido, así como la tipología y conexión con el alumnado. Tras nuestra observación comprobamos que la trascendencia de las competencias comienza en el mismo momento en el que se establecen los criterios previos de su selección, y el tipo de metodología a implementar en las correspondientes actividades no presenciales. Como primeras conclusiones, destacamos ciertas competencias indispensables, tales como la capacidad de diálogo interactivo, el dominio de los recursos audiovisuales o la especialización didáctica en contextos formativos especializados.

Palabras claves: Innovación docente, competencia didáctica, online, calidad, posgrado.

\section{Teaching skills in graduate online courses university}

\begin{abstract}
The purpose of this article, which shows the beginning of an ambitious research, is to analyze the main techniques and guidelines that require teachers in distance courses Graduate. Educational provision that begins to increase very strongly in most prestigious university institutions whose experimental basis is retained in a successful start-up and consolidation. We examined the new methods in terms of form and content, and the types and connection with students. Following our observation we found that the importance of skills starts at the same time establishing the previous criteria of choice, and type of methodology to be implemented in the corresponding non-contact activities. As early findings, we highlight certain essential practices, such as the ability of interactive dialogue, the domain of audiovisual resources or teaching expertise in specialized learning contexts.
\end{abstract}

Key Words: Teaching innovation, teaching skills, online, quality, graduate

\section{Referencia normalizada}

GONZÁLEZ CONDE, María Julia; SALGADO SANTAMARÍA, Carmen y FOMBONACADAVIECO, Javier (2012): "Competencias didácticas en cursos online de posgrado universitario". Estudios sobre el mensaje periodístico. Vol. 18, núm. especial octubre, págs.: 443-453. Madrid, Servicio de Publicaciones de la Universidad Complutense.

Sumario: 1. Introducción. 2. Metodología. 3. Resultados; 3.1. Tipología de la oferta educativa online en posgrado en las universidades públicas madrileñas. 4. Discusión. 5. Conclusiones. 6. Referencias bibliográficas. 


\section{Introducción}

La irrupción de las TIC como herramientas imprescindibles en la Sociedad de la Información, que se extiende al Conocimiento y al Aprendizaje, ha conformado en la universidades nuevas modalidades de educación no presencial, que sumadas a las ya existentes, tienden a sistematizar la incorporación de innovaciones tecnológicas docentes, en sus planes de estudio, con el fin de atender a una heterogénea demanda educativa, a una exigente formación continua, a una cada vez mayor especialización de contenidos y, por último, y en definitiva, a una adaptación adecuada a la realidad profesional. Todo un proceso dinámico y coordinado que viene a confluir en los denominados cursos de posgrado, a quienes se les pretende asignar un especial valor cualitativo. El análisis de las técnicas y competencias de los docentes en los cursos de posgrado online, serán nuestro objeto de estudio y se desprenden, en cierta medida, de los primeros resultados fruto de una ambiciosa investigación ${ }^{1}$.

Sin embargo, la delimitación de uso de las Tecnologías de la Información (TI) es una cuestión compleja dada su multiplicidad, que supera actualmente la definición básica de los instrumentos que proporcionan la adquisición, producción, almacenamiento, tratamiento, comunicación, registro y presentación de informaciones, en forma de voz, imágenes y datos contenidos en señales de naturaleza acústica, óptica o electromagnética (Cebrián de la Serna, 2005). Algunas particularidades de las TI tales como la inmaterialidad, interactividad, instantaneidad, innovación, elevados parámetros de calidad de imagen y sonido, digitalización, automatización, interconexión o diversidad, nos lleva a elegir el término "el espacio virtual", intangible, cuyo grado de inmediatez e inmaterialidad aporta beneficios sociales e implica directamente a la educación (Cabero, 1998). Falta ahora que se produzca una evolución educativa de la mano de las TIC que llegue a toda la comunidad universitaria para optimizar los recursos que nos ofrece, en gran medida virtualizados en nuestros entornos digitales de trabajo, que nos lleven a hablar de web 2.0, generación 2.0 y Universidad 2.0 (Salgado, 2012).

De este modo, a pesar de encontrarse en una fase inicial de experimentación, y aún con escasos estudios empíricos sobre su aplicación, la atención a los Mundos Virtuales 3D o MUVEs (MultiUser Virtual Environments) como herramientas y espacios de aprendizaje van incorporando de manera creciente, aunque tímida, a la educación universitaria como mecanismos que facilitan la organización de nuevas estrategias educativas, centradas, en este caso, en la metodología online.

El espacio o mundo virtual, también llamado entorno 3D inmersivo o metaverso, es definido dentro de un contexto gráfico y materializado en la pantalla del ordenador del usuario conectado. Este software que funciona mediante una aplicación específica permite recorrer e interactuar tanto con los elementos que allí se ofrecen, como con otros usuarios. La representación gráfica del propio usuario y su aspecto es configurable a voluntad y se denomina "avatar" (Rodríguez y Baños, 2011: 40).

${ }^{1}$ Proyecto competitivo de Innovación UCM: "Webdocencia II: Indicadores de Calidad para las buenas prácticas del Campus virtual y otros recursos de Internet en el desarrollo de cursos online" 
Todo un fenómeno telemático que se manifiesta a través de un espacio Web, que es a la vez conformador y transmisor de imagen de marca de la institución como una ventana abierta a la sociedad y exponente innovador representativo de su identidad corporativa. También supone un refuerzo en el activo intangible estratégico fundamental para la universidad, y facilita su diferenciación respecto a otras organizaciones competidoras (Cariotti, 2005), de ahí la necesidad de un diseño, que responda a una estructuración no sólo atractiva sino eficaz.

Si, además, nos situáramos al lado del alumnado descubriríamos en ellos un nuevo perfil o talante, adquirido como usuarios de las TIC y en entornos cada vez más dinámicos, icónicos, y adaptados a los nuevos planteamientos comunicacionales que crean los medios de comunicación social. El impacto positivo en la motivación (Warburton, 2009, 421), así como el estímulo hacia la participación (Jenkins, 2005), ha transformado el papel de estos usuarios, que ha derivado de una contemplación pasiva a una interacción más participativa (Pholke, 2007: 20).

Asimismo, el espacio virtual, no se considera presencial, sino representacional ya que simula la realidad; no se muestra cercano sino distante, ni sincrónico, sino multicrónico; y tampoco se basa en recintos espaciales delimitados con un interior, una frontera y un exterior, sino que depende de redes cuyos modos de interacción pueden estar diseminados por diversos países o dentro de los campus universitarios (Guanilo, 2008). En este nuevo contexto espacio temporal, y dentro del diseño pedagógico, los docentes virtuales deben alterar su enfoque hacia el "aprender haciendo" (Rodríguez, 2012), lo que le permitirá entonces al estudiante tener la sensación de formar parte de un entorno físico, a través de un espacio virtual y así poder involucrarse en el proceso evolutivo de autoaprendizaje bajo la realización de actividades y el control lejano de sus acciones.

El desarrollo tecnológico y la conjunción de los sistemas en red, exigen, pues, un diseño pedagógico basado en el citado modelo "aprender, haciendo", que nos acerque a una metodología más activa, centrada en el aprendiz, con mayor uso de recursos distribuidos y repartidos, de forma sincrónica y asincrónica (Mc Louglin, 2000; Barbera, 2001; Garrison y Archer, 2001; Gros, 2004).

Igualmente, este diseño pedagógico conlleva una fuerte carga en la acción tutorial para la comprensión y realización de actividades inmersas en el entorno virtual, apto para complementarse con espacios de interacción amigables y donde el software online educativo especializado (Moodle) adquiere una mayor significación. Este modelo supera las experiencias individuales hacia la construcción de actividades conjuntas y conocimiento compartido, a través del aprendizaje colaborativo (Gros, 2004). Un proceso formativo que además representa el reparto de objetivos y responsabilidades entre los distintos integrantes, ubicados en lugares distantes, al mismo tiempo que enfatiza el recurso digital como elemento mediador de apoyo en este tipo de aprendizaje. En este sentido, Barberá (2001) considerará el "aprendizaje mediado", en el diseño virtual, como una estrategia docente fundamental en la adquisición de conocimientos.

La tarea del tutor parece incrementarse a la de un experto multidisciplinar, un agente cultural integral e interactivo, y un intermediario esencial entre el saber y los 
procesos de aprendizaje de los alumnos (Jerónimo, et al., 2011: 7-8). Además, en este tipo de aprendizaje a distancia, y especialmente online, aparecen ciertos cambios significativos en la forma y lenguaje utilizados (Fombona y Mampaso, 2010: 75-77), como son:

- El predominio de lo visual y audiovisual ante lo verbal.

- El paso del papel impreso a la pantalla del ordenador como herramienta habitual de presentación de los datos.

- El diseño informativo que altera la linealidad del texto para convertirse en una variedad de hipertextos o hipermedia, marcando una connotación distinta a la proporcionada por la expresión lineal pausada y sistemática del texto escrito y a las disertaciones presenciales en el aula.

- El alumno, a través de las plataformas y redes interactivas, se convierte, además de consumidor, en creador y colaborador de contenidos.

\section{Metodología}

Actualmente en España el universo de estudios universitarios de posgrado, no presenciales, supera la cifra de 175 titulaciones diferentes, mientras que el número se reduce considerablemente (17) cuando éstos son ofertados por centros no universitarios, como por ejemplo el Consejo Superior de Investigaciones Científicas (CSIC) y otros institutos y sedes de ámbito internacional. En nuestro caso, hemos realizado un análisis descriptivo de la oferta educativa universitaria online ofrecida durante el curso académico 2011-12, en el nivel educativo de posgrado, centrándonos, como muestra representativa, en el ámbito de las universidades públicas de la comunidad madrileña.

Como hipótesis de trabajo observamos la existencia de nuevas prácticas didácticas vinculadas a los soportes comunicacionales telemáticos. Estrategias que vienen transformando de algún modo las actividades educativas tradicionales, provocando variaciones sustanciales en la metodología $\mathrm{y}$, por ende, configurando la educación a distancia, online, hacia un nuevo enfoque o planteamiento pedagógico.

Las hipótesis secundarias cuestionan el nuevo papel de los espacios, tiempos y procedimientos implementados en el marco presencial de las instituciones educativas universitarias; condicionantes que deberían contextualizar y delimitar las facultades y potencialidades del docente virtual.

Con ello determinamos como objetivo principal la definición de las competencias docentes necesarias para la interacción educativa de cursos de posgrado universitario, que se vinculan y/o apoyan en entornos virtuales y digitalizados.

\section{Resultados}

Los miembros del equipo de investigación han realizado una serie de acciones para el análisis de la información contenida en las webs de las universidades públicas madrileñas, que quedan resumidas en el siguiente esquema:

- Identificación de las plataformas institucionales y propias de cada una de las titulaciones.

- Definición de las categorías objeto de análisis.

- Empleo de recursos digitales que favorezcan la interacción entre los usuarios. 
Para conseguir estos fines, tomamos como punto de partida de exploración el periodo comprendido entre julio y septiembre de 2012, cuyo marco comparativo y de acción se desarrollará de forma más ampliada en sucesivas investigaciones.

\subsection{Tipología de la oferta educativa online en posgrado en las universidades pú- blicas madrileñas}

Entre las titulaciones online que ofrecen las Universidades públicas de la Comunidad de Madrid es interesante destacar que el 33,3\% de los estudios de posgrado se refiere principalmente a materias de economía y gestión empresarial; y que un $28,8 \%$ se orientan al ámbito sanitario. Por otro lado, un $20 \%$ de estos estudios está relacionado con el propio ámbito educativo, tanto en su aspecto social como docente. En líneas generales, y en la actualidad, el $80 \%$ de la oferta de cursos de posgrado online se concentra en estas tres grandes áreas de conocimiento: economía y empresa, sanidad y educación.

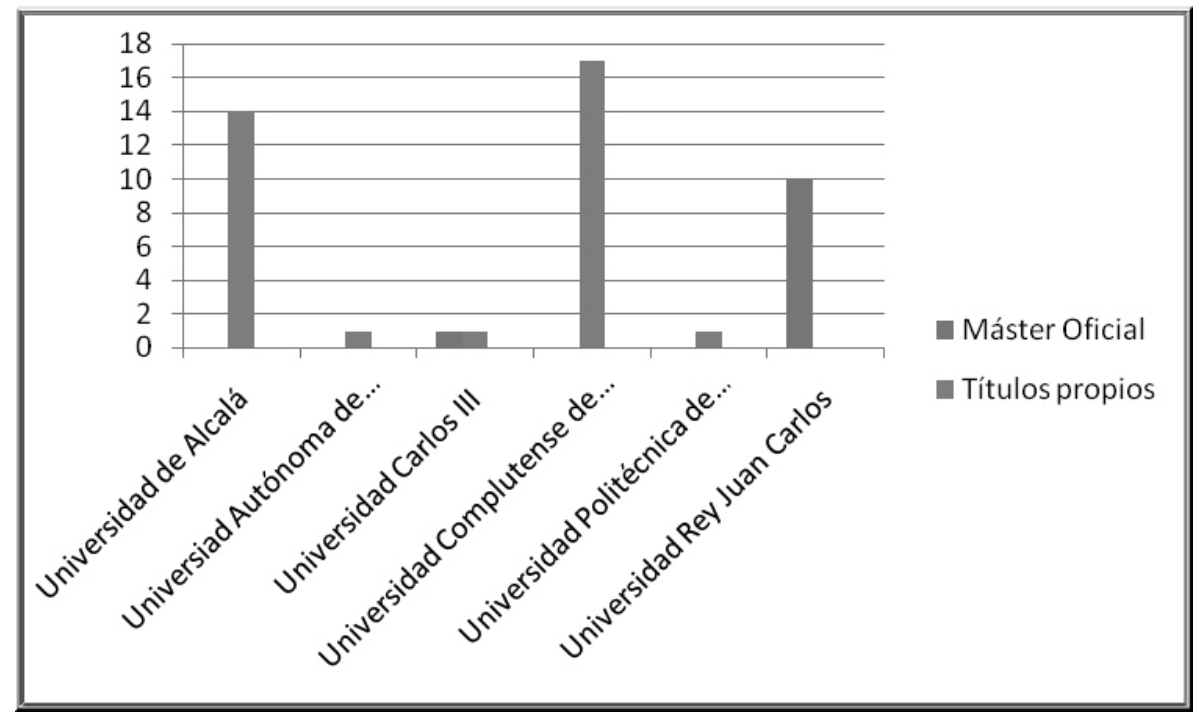

Dos de las universidades madrileñas, la Complutense y la de Alcalá de Henares, concentran la mayor parte de la oferta de cursos online de posgrado, en los Títulos Propios. Mientras que la Universidad Rey Juan Carlos lo hace en los Grados y los Másteres Oficiales; por su parte, la Universidad Autónoma ofrece un único Título propio en esta modalidad: Experto en actualización en Enfermería Familiar y Comunitaria, y la Universidad Carlos III reparte un único curso online para cada una de las titulaciones de posgrado, Título propio y Máster oficial.

La Universidad Politécnica de Madrid sólo cuenta con una oferta formativa online, o Título propio, que califica como Máster en Dirección y Gestión de proyectos software. Modalidad a distancia. 


\section{Discusión}

A lo largo de toda esta investigación se ha tenido presente el objetivo fundamental de cualquier proceso educativo que es otorgar al estudiante una formación global y eficiente. El alumnado cuenta con un número limitado de cursos de posgrado de esta modalidad mientras que no se incrementan las ofertas formativas multidisciplinares y plurimetodológicas, a pesar del refuerzo considerable en el uso mediador de los recursos digitales. Esta formación online, cuyas fórmulas, a través de Internet, mejoran la comunicación sincrónica (chats o video conferencias) y asincrónica (tutorías, foros o e-mails) con los estudiantes, y dotan de la máxima calidad y eficacia a este método de estudio, son una tercera vía de elección formativa para los alumnos.

Se trata de una labor ardua que obliga a ciertos mecanismos de filtrado, que controle la entrada ilimitada a bibliotecas de textos, imágenes, animaciones, sonidos, diagramas, ilustraciones y todo tipos de datos que constituyen la "metanarrativa intertextual", capaz de generar significaciones diferentes para cada lectura posible" (González Conde, 2009: 344)

Del mismo modo, el diseño de la oferta educativa se enriquece con la opción de una modalidad formativa no presencial, y es cuando el docente virtual debe gestionar muy bien su actividad, para que sirva de orientación y ayuda en el proceso mediador entre la información y el alumnado; un rasgo que le confiere una labor diferente a la llevada a cabo hasta ese momento; y que, indudablemente, representa una mayor complejidad, ya que exige una máxima planificación, previsión, conocimiento y una atención continuada. Asimismo, además de aplicar las nuevas tecnologías a la educación, deberá diseñar otros escenarios y tiempos donde el estudiante pueda colaborar y participar en su aprendizaje.

Ya Bork (1986) exponía los requisitos y el potencial de los equipos informáticos en el proceso de enseñanza-aprendizaje, si se respetaban las siguientes funciones:

- Enseñanza interactiva a través de un diálogo continuo.

- Máxima individualización tanto desde el punto de vista de la adaptación en la presentación de contenidos, insistiendo en una "humanización" del proceso.

- Posibilidad en la experimentación de conocimientos, no factibles en el sistema de enseñanza presencial.

- Supervisión y control del proceso de aprendizaje, permitiendo una orientación precisa en cada momento.

- Comprobación de tiempo y secuencia de la presentación del material didáctico, formal y práctico.

- Control sobre el contenido, que permita a sus componenetes disponer de una situación más racional y global.(Ortiz, Rodríguez y Pérez, 2011)

- Superación de factores humanos que interfieren en los procesos de aprendizaje que podrían ser excluidos por una presentación impersonal de la información.

Igualmente, coincidimos con Ivars y Jurado (2011: 215) al indicar que estos recursos trasforman el proceso de enseñanza y aprendizaje, según los siguientes puntos: 
- Desarrollo de habilidades de búsqueda, selección, presentación, clasificación, reflexión y crítica de la información ofrecida en cada una de las unidades didácticas, materias o temas tratados.

- Adaptación y presentación de recursos audiovisuales y formas icónicas.

- Desarrollo de la capacidad creativa y creadora de materiales didácticos empleados.

- Desarrollo de iniciativas que promueven el intercambio de información.

- Trabajo orientado sobre contenidos más novedosos y dinámicos.

- Desarrollo de un sentido de responsabilidad, pertenencia y colaboración en un nuevo entorno educativo.

Los docentes de cursos universitarios de posgrado, de cualquier tipo de modalidad de educación a distancia, con soporte en Internet, precisan utilizar un diseño pedagógico que permita intervenir en técnicas experimentales, más conveniente y adecuado para estudiantes adultos, cuyo marco teórico, desde la aplicación informativa al conocimiento, pueda confrontarse con su aplicación, en la tarea cotidiana, para alcanzar la concepción de aprendizaje significativo. Una práctica consolidada en el ciberperiodismo (Zamarra, 2002)

No olvidemos tampoco la necesidad de adaptación a los desarrollos técnicos, hasta el punto de hacer que la realidad pueda ser enriquecida con las experiencias virtuales; tal es el caso de los desarrollos de "Realidad Aumentada", una acomodación a nuevos recursos, los dispositivos portátiles, que han vuelto ubicuo el espacio donde tendrá lugar el proceso de enseñanza y aprendizaje (Fombona, et al., 2012: 207-218).

Los estudios coinciden en precisar que este tipo de enseñanza requiere la selección de entornos virtuales que contribuyan a la creación de comunidades de aprendizaje y de actividad práctica. Espacios educativos que puedan dotar a sus participantes de las herramientas imprescindibles para la comunicación virtual, el diálogo y la interacción, tanto a través de audio, texto, o del apoyo visual con elementos icónicos, lo que reforzaría la acción del tutor. Ciertos recursos virtuales que tienden a favorecer la comunicación con el profesor, además de crear un nuevo espacio interactivo comunitario, como son los foros, que se habilitan en las plataformas de las asignaturas. (Berná y Fernández, 2012)

En esta línea, la incorporación de las tecnologías de la "Realidad Virtual Inmersiva" (RVI) permite la experimentación de nuevas formas de trasmitir la información, donde podrían aparecer problemas de comprensión práctica no percibida por el docente, o el desarrollo de habilidades del estudiante en cuestiones más profesionales relacionadas con la materia de la que se trate, todo ello como valor añadido al propio proceso educativo (González y Chávez, 2011).

El entorno inmersivo parece ofrecer una serie de elementos útiles, capaces de romper las barreras existentes en la confrontación estudiante-tutor. Por un lado parece que existe un atractivo derivado de su componente lúdico (inmersión en el metaverso), y por otro, motiva la propia interacción en tiempo real y en unas condiciones semejantes a las de la realidad. Este argumento es apoyado por Rodríguez y Baños (2011) que destacan el impacto generado por la creación de una identidad y su proyección a tra- 
vés de un avatar, que supone una paradoja, difícil de entender, por la sensación de anonimato y al mismo tiempo de presencia real como sujeto.

También en esta línea, Castronova (2001) define los mundos virtuales con tres rasgos fundamentales:

1. Interactividad: porque el programa existe en un equipo con conexión a Internet, que admite el acceso remoto y simultáneo de un gran número de personas. Funciona como un espacio electrónico de actividades percibidas y compartidas por multitud de usuarios.

2. Corporeidad: cuando los usuarios acceden al programa a través de un interfaz, que simula un entorno físico, donde entrar, observar e interactuar.

3. Persistencia: el programa sigue funcionando libremente de forma autónoma, con independencia de los usuarios que estén o no conectados

Los datos nos indican que la aplicación de las TIC a través de redes implica un incremento de la oferta educativa pero no una intensificación en la calidad de enseñanza, dado que en ocasiones sólo se traduce en una transposición de los planteamientos pedagógicos tradicionales que persisten junto a nuevas formas de organización y representación de la información habitual. Se hace necesario, por tanto, discutir y valorar esas nuevas acepciones de acuerdo a la consecución de una calidad educativa y a unas mejoras en las condiciones de la mediación didáctica, que según González y Chávez (2011) no radica sólo en la forma del medio o el soporte utilizado sino en su adecuación curricular a unos objetivos previstos, a unos contenidos adecuados y a una metodología correcta. Los avances significativos en la calidad se relacionan con la suma de un todo integrado de los nuevos elementos y modelos educativos adecuados a sus características (García Aretio, 2007) que además [...] exigen nuevas competencias para el ejercicio de las profesiones y para la práctica educativa de la universidad española que, por un lado nunca deberá perder su esencia del conocimiento de la Ciencia, y por otro tampoco puede quedarse impasible ante el proceso de innovación de casi todo lo que nos rodea" (Flores y Esteve, 2010)

\section{Conclusiones}

Es necesario atraer al alumnado hacia una nueva concepción educativa, predispuesta a la utilización de plataformas virtuales, abiertas, que cuenten con determinadas peculiaridades:

- Búsqueda de un alumnado heterogéneo en conocimientos, aptitudes y actitudes de aprendizaje y estudio. Se trata de una plataforma flexible y de un procedimiento abierto, de construcción constante, que se adaptará a las necesidades de cada alumno y a los resultados obtenidos.

- Posibilidad de compaginar estudios y trabajo, lo que debe tenerse en cuenta en relación con la asistencia más o menos frecuente en las tutorías, evolución y disposición temporal.

- Proceso cercano para conseguir implicación y sentido de pertenencia a esa comunidad educativa.

- También es adecuado dotar la oferta formativa de valor y significado práctico así como de utilidad profesional directa. Las tecnologías crean nuevos escenarios 
de aprendizaje que las universidades deben incorporar y sus docentes convertirse en subsidiarios de tal innovación. Pero, para ello, deben establecerse claras normas y pautas de conducta que obedezcan a un adecuado diseño pedagógico, afín al programa marcado y adaptado a la comunidad de aprendizaje, a la que se dirige.

\section{Referencias bibliográficas}

BARBERÁ, Elena; BADIA, Antoni; y MOMINÓ, Josep Ma . (Coords., 2001): La incógnita de la educación a distancia; Barcelona, Paidós.

BERNÁ SICILIA, Celia y FERNÁNDEZ CASTRILLO, Carolina (2012): "La comunicación entre profesores y estudiantes de periodismo: canales y espacios de interacción en entornos online de aprendizaje" en Revista de Comunicación Vivat Academia $\mathrm{n}^{\mathrm{o}}$ 117E, Febrero 2012. Madrid, Universidad Complutense de Madrid. http://www.ucm.es/info/vivataca/numeros/n117E/DATOSS.htm

BORK, Alfred (1986): El ordenador en la enseñanza. Análisis y perspectivas de futuro. Barcelona: Gili.

CABERO, Julio (1998): Las aportaciones de las nuevas tecnologías: Reflexiones para comenzar el debate. Madrid, UNED.

CASTRONOVA, Edward (2001): "Virtual Worlds: A First-Hand Account of Market and Society on the Cyberian Frontier" en Cesifo Working Paper Series $n^{\circ}$ 618: http://ssrn.com/abstract=294828 [fecha de consulta: 1 de abril de 2012].

CEBRIÁN DE LA SERNA, Manuel (coord., 2005): Tecnologías de la información y comunicación para la formación de docente. Madrid, Pirámide.

GARCÍA ARETIO, Lorenzo. (Coord., 2007): De la educación a distancia a la educación virtual. Barcelona, Ariel.

FLORES VIVAR, Jesús y ESTEVE RAMÍREZ, Francisco (Editores, 2010): BlogUniversidad. Los blogs en la Universidad como iniciativas prácticas de innovación docente. Análisis, evolución y tendencias. Madrid, Servicio de Publicaciones de la Universidad Complutense.

FOMBONA, Javier y MAMPASO, Joanne (2010): "Influencia de las TICS Audiovisuales en el nuevo perfil de alumnado". EduPsykhé. Revista de Psicología y educación, $\mathrm{n}^{\mathrm{o}}$ 9(1), pp. 61-83.

FOMBONA, Javier; PASCUAL, Angeles y AMADOR, Filomena (2012): "Realidad Aumentada, una evolución de las aplicaciones de los dispositivos móviles". PixelBit. Revista de Medios y Educación, nº41, pp.: 207-220.

GONZÁLEZASPERA, Alma Lilia y CHÁVEZ HERNÁNDEZ, Gerardo (2011): “La realidad virtual inmersiva en ambientes inteligentes de aprendizaje. Un caso en la educación superior". Revista Icono14, Año 9, Volumen 2. pp. 122-137. En: http://www.icono14.net [fecha de consulta: 1 de abril de 2012]. 
GONZÁLEZ CONDE, María Julia (2009): "Interactividad a toda costa". En FLORES, Jesús Miguel y ESTEVE, Francisco: Periodismo Web 2.0. Madrid, Fragua; pp. 341-347

GROS, Begoña. (2004): La construcción del conocimiento en la red: límites y posibilidades. Revista Electrónica Teoría de la Educación: Educación y Cultura en la Sociedad de la Información. En: http://campus.usal.es/ teoriaeducacion/rev_numero_05/n5_articulos.htm [fecha de consulta: 1 de mayo de 2012].

GUANILO, Santos (2008): La educación virtual y el constructivismo social. Tacna, Perú, Fatla.

IVARS, Begoña y JURADO, Montserrat (2011): "Innovación didáctica digital en los contenidos fundamentales de la Licenciatura de Periodismo", en Flores Vivar, Jesús (coord.): Reinventar el Periodismo y los medios. Madrid, Fragua, pp. 215-222.

JENKINS, Henry (2005): "Getting into the gamel" in Educational Leadership, n 62 (7).

JERÓNIMO MONTES, José Antonio; ANDRADE CORTÉS, Lidia del Carmen y ROBLES MELGAREJO, Ascensión (2011): "El diseño educativo en los mundos virtuales. La curva de aprendizaje inmersito". Revista Icono14, Año 9, $n^{\circ}$ 2. pp. 21-38. En: de http://www.icono14.net [fecha de consulta: 13 de marzo de 2012].

ORTIZ SOBRINO, Miguel Ángel; RODRÍGUEZ BARBA, Dolores; y PÉREZ SERRANO, María José (2011): "Perfil de entrada de los futuros alumnos de comunicación en las universidades de Madrid, en 2010/2012: la reafirmación de los rasgos", en Revista de Comunicación SEECI n²6, noviembre. Madrid, Universidad Complutense de Madrid. http://ucm.es/info/seeci/Numeros/Numero/26 /DATOS.html [fecha de consulta: 27 de mayo de 2012]..

PHOLKE, Alfred (2007): Second Life as an emerging platform for intercultural education. Unpublished. Masters Dissertation. Berlin, Freie Universität.

RODRÍGUEZ GARCÍA, Teresa y BAÑOS GONZÁLEZ, Miguel (2011): "E-learning en mundos virtuales 3D. Una experiencia educativa en Second Life". Revista Icono14, Año 9, $n^{\circ}$ 2. pp. 39-58. En: http://www.icono14.net [fecha de consulta: 1 de marzo de 2012].

SALGADO, Carmen (2012): "EL EEES, un destino seguro a distintas velocidades", en El reto en la innovación docente: El EEES como punto de encuentro. Madrid, Editorial Visión Libros, pp. 205-237.

ZAMARRA LÓPEZ, Mercedes (2002): "El periodismo digital y la nueva formación ciberperiodística", en Revista de Comunicación SEECI n⿳ 9, noviembre 2002. Madrid, Universidad Complutense de Madrid. http://ucm.es/info/seeci/Numeros/Numero/9/DATOS.html [fecha de consulta: 3 de junio de 2012].

WARBURTON, Steven (2009): "Second Life in Higher Education: Assessing the Potential for and the Barriers to Deploying Virtual Worlds in Learning and Teaching", en British Journal of Educational Technology, nº 40. 


\section{María Julia GONZÁLEZ CONDE}

mariajul@ccinf.ucm.es;

Universidad Complutense, Facultad de Ciencias de la Información, Avda. Complutense, s/n. 28040 Madrid

\section{Carmen SALGADO SANTAMARÍA}

carmen.salgado@ccinf.ucm.es

Universidad Complutense, Facultad de Ciencias de la Información, Avda. Complutense, s/n. 28040 Madrid

Directora Dpto. Periodismo II

\section{Javier FOMBONA CADAVIECO}

fombona@uniovi.es

Universidad de Oviedo, Facultad de Formación del Profesorado y Educación, Aniceto Sela, s/n, 33005 Oviedo

Vicedecano de Coordinación Académica 